\title{
APPLICATION OF GRADIENT STEEPEST DESCENT METHOD TO THE PROBLEM OF CRYSTAL LATTICE PARAMETRIC IDENTIFICATION
}

\author{
A.S .Shirokanev ${ }^{1}$, D.V. Kirsh ${ }^{1,2}$, A.V. Kupriyanov ${ }^{1,2}$ \\ ${ }^{1}$ Samara National Research University, Samara, Russia \\ ${ }^{2}$ Image Processing Systems Institute - Branch of the Federal Scientific Research Centre "Crys- \\ tallography and Photonics" of Russian Academy of Sciences, Samara, Russia
}

\begin{abstract}
The objective of this paperwork is the development of a crystal lattice parameter identification algorithm, which allows obtaining a more accurate solution compared to the Bravais unit cell estimation algorithm. To achieve the objective, we suggest solving the parameter identification problem using the steepest descent gradient method. The study of the parameter identification accuracy was conducted on a large number of modeled crystal lattices using the edges and angles similarity measures for Bravais unit cells.
\end{abstract}

Keywords: crystal lattice, Bravais unit cell, Wigner-Seitz unit cell, similarity measure, translation vector, parameter identification, gradient steepest descent method.

Citation: Shirokanev AS, Kirsh DV, Kupriyanov AV. Application of gradient steepest descent method to the problem of crystal lattice parametric identification. CEUR Workshop Proceedings, 2016; 1638: 393-400. DOI: 10.18287/1613-0073-2016-1638-393-400

\section{Introduction}

Nowadays, much attention has been concentrated on reconstruction of threedimensional objects $[1,2,3]$. In particular in crystallography, reconstruction of a three-dimensional crystal lattice structure is related directly to a parameter identification problem, which is one of the basic problems of X-ray diffraction analysis $[4,5]$. Unfortunately, such methods either lack sufficient accuracy or fail to describe comprehensively the crystal properties [4, 6-10]. The majority of universal methods aimed at the solution of the parameter identification problem with high accuracy are based on estimating parameters of a unit cell [6-8].

The most well-known crystal lattice model was offered by Auguste Bravais. The Bravais model is based on unit cell representation: the entire lattice can be constructed by translation of a single cell. All unit cells are divided into seven lattice systems according to edge lengths and angle values (Fig. 1) [11]. 
With evolving technology, the parameter identification algorithms as well as the crystal lattice comparison methods have become more relevant $[4,7-10,12,13]$.

The objective of a crystal lattice parameter identification method is to estimate unit cell parameters. There are several methods that offer a solution to the problem: NIST lattice spacing comparator [14], parameter identification methods based on estimation of atomic packing factor [15] and distances between isosurfaces [16]. However, these methods are not universal and have a number of disadvantages, such as strong dependence between the crystal lattice identification accuracy and the lattice system, high sensitivity to distortions of crystal lattice point coordinates or complexity of the sample preparation.
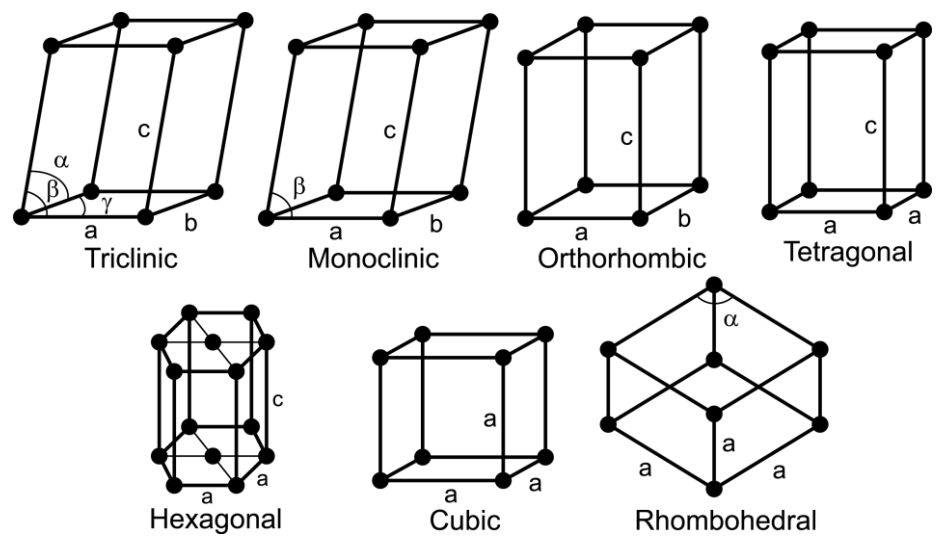

Fig. 1. Unit cells of seven lattice systems

Among the existing universal methods that provides high accuracy of crystal lattice parameter identification, we can distinguish the following ones: "The lattice identification method based on estimation of Bravais unit cell parameters" [8], "The lattice identification method based on estimation of the Wigner-Seitz cell volumes" [8]. In the experimental section, the comparison of the results obtained by these methods and the developed ones are presented.

\section{The lattice identification method based on estimation of Bravais unit cell parameters}

The method is based on calculation of six key parameters of the Bravais unit cell, i.e. three edge lengths and three included angles [6, 7, 9, 11, 19].

Initial data for the identification method based on estimation of the Bravais unit cell parameters are a finite set of radius-vectors of crystal lattice nodes.

The identification method involves a search of three non-coplanar vectors in the original set of nodes: the first vector has a minimum norm; the second vector does not lie on a straight line with a directing vector equal to the first vector; the third vector does not lie on a plane made by two found vectors. 


\section{The lattice identification method based on estimation of Bravais unit cell parameters}

The method is based on calculation of six key parameters of the Bravais unit cell (Fig. $2)$, i.e. three edge lengths and three included angles $[6,8,10]$.

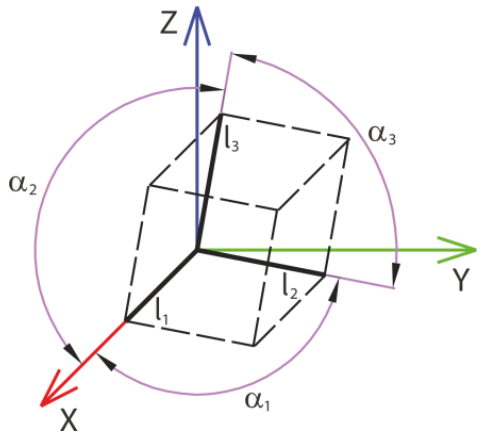

Fig. 2. Bravais unit cell

Initial data for the identification method based on estimation of the Bravais unit cell parameters are a finite set of radius-vectors of crystal lattice nodes.

The identification method involves a search of three non-coplanar vectors in the original set of nodes: the first vector has a minimum norm; the second vector does not lie on a straight line with a directing vector equal to the first vector; the third vector does not lie on a plane made by two found vectors.

The lattice identification method based on estimation of the Wigner-Seitz cell volumes

In order to find volumes of the Wigner-Seitz cells (Fig. 3), we must construct planes limiting the cell and estimate its volume using the Monte-Carlo method - a random scattering of a large number of points into a lattice area and calculation of a number of points fell into the limited area of the Wigner-Seitz cell [8].

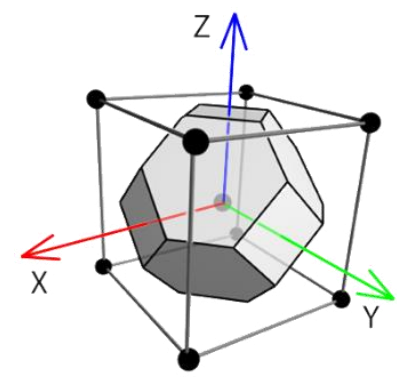

Fig. 3. Wigner-Seitz unit cell 
Initial data for the identification method based on estimation of Wigner-Seitz cell volumes are the $P$ - number of entered points and the set of radius-vectors of crystal lattice nodes as follows:

$X=\left\{\bar{x}_{l}\right\}_{l=1}^{L}, \bar{x}_{l}=\left(\bar{x}_{l 1}, \bar{x}_{l 2}, \bar{x}_{l 3}\right)^{\mathrm{T}} \in \mathbb{R}^{3}$.

The identification method involves calculation of Wigner-Seitz cell volumes by constructing the limiting planes: $p_{l 1} x+p_{l 2} y+p_{l 3} z-\left\|\bar{p}_{l}\right\|^{2}=0,1 \leq l \leq L-1$; generating $L$ - values of three-dimensional random vectors which are uniformly distributed in the whole lattice volume and counting the number of vectors that hit in the region limited by planes. Calculation of the cell volume is based on the fact that the probability of hit in the Wigner-Seitz cell region is proportional to its measure (volume).

In the presented work, we use only information about limiting planes to reconstruct the whole lattice. It is necessary to compare all developed parameter identification methods uniformly.

\section{Crystal lattice parameter identification algorithm based on optimizing translation vector search using the gradient steepest descent method}

In the context of this paperwork, we have developed a crystal lattice parameter identification algorithm based on the steepest descent gradient method. The fundamental element of this algorithm is the Bravais lattice model described by three translation vectors $\bar{a}_{1}, \bar{a}_{2}$ and $\bar{a}_{3}$ [17]. The set of lattice nodes is expressed as:

$X=\left\{x=i \bar{a}_{1}+j \bar{a}_{2}+k \bar{a}_{3}\right\}: i, j, k \in \mathbb{Z}$.

In this case, both algorithms shall require initial approximation as an additional input parameter. Particularly, the result vectors of the lattice identification method based on estimation of Bravais unit cell parameters can be used as the initial approximation.

The objective function of optimization is as follows:

$$
E\left(\bar{a}_{1}, \bar{a}_{2}, \bar{a}_{3}\right)=\sum_{l=1}^{L} \min _{i, j, k}\left\|\bar{x}_{l}-\left(i \bar{a}_{1}+j \bar{a}_{2}+k \bar{a}_{3}\right)\right\|^{2},
$$

where $L$ is the number of nodes in the lattice.

Let us introduce the following notation:

$$
\begin{aligned}
& \mathrm{A}=\left(\begin{array}{lll}
\bar{a}_{1} & \bar{a}_{2} & \bar{a}_{3}
\end{array}\right) \in \mathbb{R}^{3 \times 3} ; \\
& \bar{n}_{l}=\left(\begin{array}{lll}
i_{l} & j_{l} & k_{l}
\end{array}\right)^{T} ; \\
& \mathrm{N}_{l}=\bar{n}_{l} \bar{n}_{l}^{T} ; \\
& \bar{w}_{l}^{s}=i_{l} \bar{a}_{1}^{s}+j_{l} \bar{a}_{2}^{s}+k_{l} \bar{a}_{3}^{s}-\bar{x}_{l},
\end{aligned}
$$


where $s$ is the step number in the descent.

In this case a gradient (2) and a descent factor (3) are as follows:

$$
\begin{aligned}
& \nabla E(\mathrm{~A})=2\left[\mathrm{~A} \cdot \sum_{l=1}^{L} \mathrm{~N}_{l}-\sum_{l=1}^{L} \bar{x}_{l} \bar{n}_{l}^{T}\right] ; \\
& \lambda_{s}=\frac{\sum_{l=1}^{L}\left(\bar{w}_{l}^{s}, \nabla E\left(\mathrm{~A}^{s}\right) \bar{n}_{l}\right)}{2 \sum_{l=1}^{L}\left\|\nabla E\left(\mathrm{~A}^{s}\right) \bar{n}_{l}\right\|^{2}} .
\end{aligned}
$$

Expressions (2) and (3) are recorded in compact form. The desired solution is the matrix (triple translation vectors). Figure 4 shows the convergence of translation vectors to solution for a two-dimensional lattice.

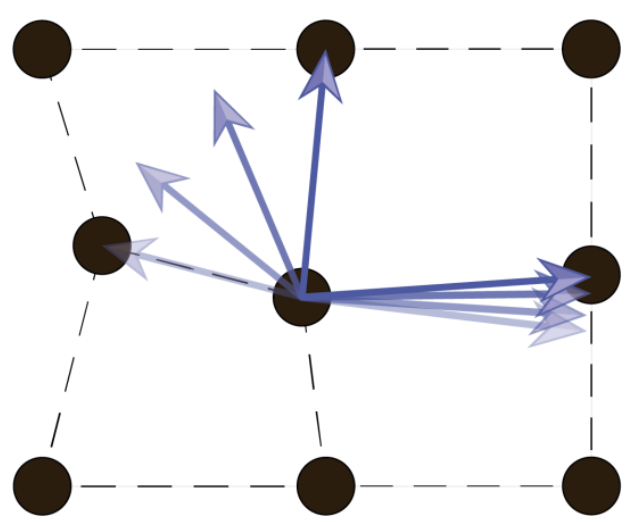

Fig. 4. Convergence of translation vectors to the solution

\section{Analysis of parameter identification accuracy of the developed algorithms}

The aim of the crystal lattice parameter identification algorithm based on the steepest descent gradient method is finding the vectors $\bar{a}_{1}, \bar{a}_{2}$ and $\bar{a}_{3}$, minimizing the objective function (1). In addition, the objective function (1) determines the error of the obtained solution in relation to the source set of nodes.

Using computational experiments, we studied the dependence between the reduction of the objective function (1) and the lattice systems. For the experiments, 20 lattices were generated for each crystal system. All modeled lattice consisted of 125 nodes (5 translations in each direction). Then the lattices was distorted through a random offset of each node from its ideal position in a random direction at a distance no more 
than $0.5 \AA$. The solution error (1) between the original lattice and the lattice reconstructed by estimated parameters determined the accuracy of parameter identification methods. Figure 5 shows the obtained results as values of the averaged objective function.

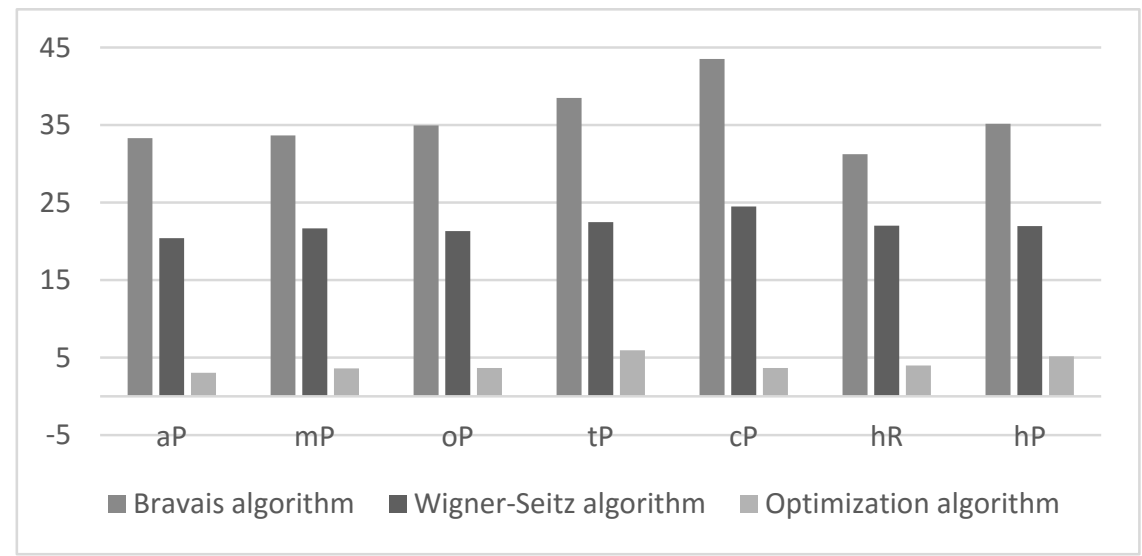

Fig. 4. Results of study of dependence of solution error from syngony when running parameter identification algorithms being tested

According to experiment results, the Bravais unit cell parameter estimation algorithm is highly sensitive to the distortion of lattice structure. It is obvious, since the method analyze only 4 nearest lattice nodes but not the whole lattice structure. On the contrary, a Wigner-Seitz cell of three-dimensional lattice are described by 27 lattice nodes. As a result, the Wigner-Seitz cell volume estimation algorithm demonstrated significantly better results than the previous one. The error decreased on average by $47 \%$. However, the Wigner-Seitz cell volume estimation algorithm has a crucial disadvantage - an extremely high computational complexity.

The best result was obtained using the developed parameter identification algorithm based on the gradient steepest descent method. It greatly improved the results obtained by the Bravais unit cell parameter estimation algorithm and reduced almost sixfold the solution error.

\section{Conclusion}

In the presented work, we have developed algorithm of parameter identification based on the gradient steepest descent method. The algorithm uses the result vectors of the lattice identification method based on estimation of Bravais unit cell parameters is used as the initial approximation.

The experiments proved that the parameter identification algorithm based on the gradient steepest descent method is stable to lattice distortion and allows to reduce six- 
fold the solution error compared to the Bravais unit cell parameter estimation algorithm and threefold compared to the Wigner-Seitz cell volumes estimation algorithm.

\section{Acknowledgments}

This work was partially supported by the Ministry of education and science of the Russian Federation in the framework of the implementation of the Program of increasing the competitiveness of SSAU among the world's leading scientific and educational centers for 2013-2020 years; by the Russian Foundation for Basic Research grants (\# 14-07- 97040, \# 15-29- 03823, \# 15-29- 07077, \# 16-57- 48006); by the ONIT RAS program \# 6 "Bioinformatics, modern information technologies and mathematical methods in medicine" 2016.

\section{References}

1. Fursov VA, Goshin YeV. Information technology for digital terrain model reconstruction from stereo images. Computer Optics, 2014; 38(2): 335-342. [in Russian]

2. Kotov AP, Fursov VA, Goshin YeV. Technology for fast 3d-scene reconstruction from stereo images. Computer Optics, 2015; 39(4): 600-605 [in Russian]. DOI: 10.18287/01342452-2015-39-4-600-605.

3. Kudinov IA, Pavlov OV, Kholopov IS. Implementation of an algorithm for determining the spatial coordinates and the angular orientation of an object based on reference marks, using information from a single camera. Computer Optics, 2015; 39(3): 413-415 [in Russian]. DOI: 10.18287/0134-2452-2015-39-3-413-419.

4. Shirokanev AS, Kirsh DV, Kupriyanov AV. Researching methods of reconstruction of three-dimensional crystal lattice from images of projections. CEUR Workshop Proceedings, 2015; 1490: 290-297. DOI: 10.18287/1613-0073-2015-1490-290-297.

5. Kharitonov SI, Volotovskiy SG, Khonina SN, Kazanskiy NL. A differential method for calculating x-ray diffraction by crystals: the scalar theory. Computer Optics, 2015; 39(4): 469-479 [in Russian]. DOI: 10.18287/0134-2452-2015-39-4-469-479.

6. Kupriyanov AV, Kirsh DV. Estimation of the Crystal Lattice Similarity Measure by Three-Dimensional Coordinates of Lattice Nodes. Optical Memory \& Neural Networks (Information Optics), 2015; 24(2): 145-151.

7. Kirsh DV, Kupriyanov AV. Crystal lattice identification by coordinates of their nodes in three dimensional space. Pattern recognition and image analysis, 2015; 25(3): 456-460.

8. Kirsh DV, Kupriyanov AV. Identification of Three-Dimensional Crystal Lattices by Estimation of Their Unit Cell Parameters. CEUR Workshop Proceedings, 2015; 1452: 40-45.

9. Soldatova OP, Lyozin IA, Lyozina IV, Kupriyanov AV, Kirsh DV. Application of fuzzy neural networks for defining crystal lattice types in nanoscale images. Computer Optics, 2015; 39(5): 787-794 [in Russian]. DOI: 10.18287/0134-2452-2015-39-5-787-794.

10. Kirsh DV, Kupriyanov AV. Modeling and Identification of Centered Crystal Lattices in Three-Dimensional Space. CEUR Workshop Proceedings, 2015; 1490: 162-170. DOI: 10.18287/1613-0073-2015-1490-162-170.

11. Hammond C. The Basic of Crystallography and Diffraction. Third Edition. Oxford University Press Inc., 2009: 84-95. 
12. Brandon D, Kaplan U. Microstructure of materials. Methods of research and monitoring. Technosphere Publisher, 2004: 39-52.

13. Lawrence CA, Herbert JB. Lattices and Reduced Cells as Points in 6-Space and Selection of Bravais Lattice Type by Projections. Acta Cryst, 1988: 1009-1018.

14. Kessler E, Henins A, Deslattes R, Nielsen L, Arif M. Precision Comparison of the Lattice Parameters of Silicon Monocrystals. Journal of Research of the National Institute of Standards and Technology, 1994; 99: 1-18.

15. Smith W. Foundations of Materials Science and Engineering. McGraw-Hill, 2004: 67-107.

16. Patera J, Skala V. Centered cubic lattice method comparison. 17th ALGORITMY Conference on Scientific Computing, 2005: 309-319.

17. Shaskolskaya M.P. Crystallography: Manual for Institutes of Higher Education. Higher School, 1984: 10-14. [in Russian] 